Document downloaded from:

http://hdl.handle.net/10251/44429

This paper must be cited as:

Gahete Arias, C.; Baquero Escudero, M.; Valero-Nogueira, A.; Vila Jiménez, A. (2013). Test-Fixture for Suspended-Strip Gap-Waveguide Technology on Ka-Band. IEEE Microwave and Wireless Components Letters. 23(6):321-323.

doi:10.1109/LMWC.2013.2258000.

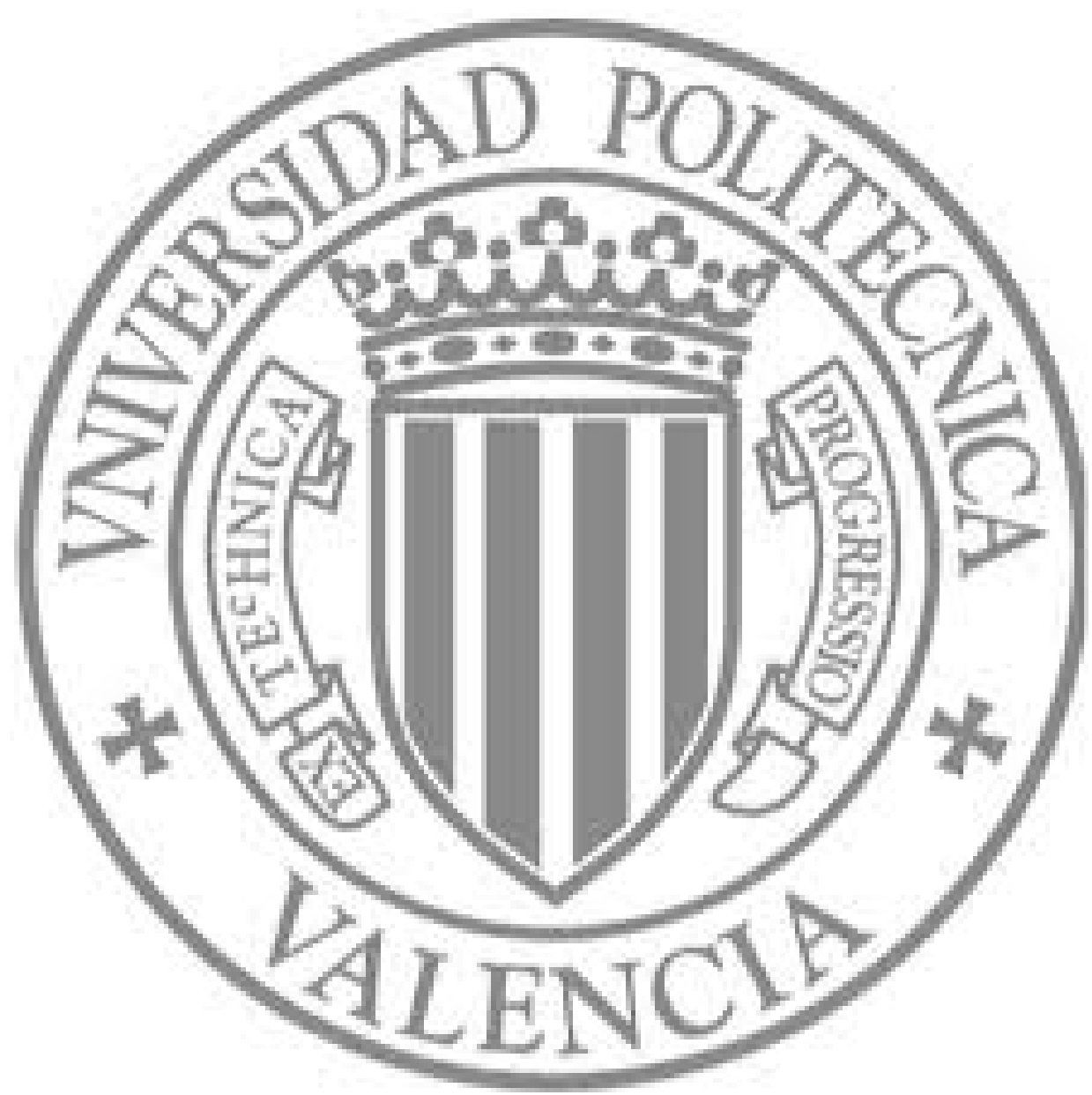

The final publication is available at

http://dx.doi.org/10.1109/LMWC.2013.2258000

Copyright Institute of Electrical and Electronics Engineers (IEEE) 


\title{
Test-Fixture for Suspended-Strip Gap-Waveguide Technology on Ka-band
}

\author{
Carlos Gahete Arias, Student Member, IEEE, Mariano Baquero Escudero, Member, IEEE, \\ Alejandro Valero Nogueira, Senior Member, IEEE, Antonio Vila Jiménez
}

\begin{abstract}
This paper presents a Test-Fixture for a SuspendedStrip Gap-Waveguide technology that provides a fast measurement system for the prototyping stage. For this purpose, a transition from standard rectangular waveguide to this new technology has been developed. A TRL kit and a repeatability study have been made to ensure the reliability of the process, and several straightforward circuits have been manufactured and measured.
\end{abstract}

Index Terms - Test-Fixture, Millimeter-Wave, Ridge GapWaveguide.

\section{INTRODUCTION}

A new transmission line for millimeter and sub-millimeter waves, called gap-waveguide, has recently been proposed [1] - [2]. Experimental verification and several designs of microwave circuits were presented in [3]. The contact between the parallel metal parts is not required. Furthermore, because of the absence of dielectric, a low loss has been demonstrated for the Ridge Gap-Waveguide (RGW) [4]. However, the geometry for the RGW represents a drawback from the point of view of manufacturing. The use of a metal ridge for the confinement and guiding of the electromagnetic fields involves the rearrangement of the bed of nails for each circuit. This can result in excessive time consumption and excessive costs, especially in the prototype stage.

An alternative practical realization based on a suspended strip on a bed of nails was presented in [5]. This configuration replaces the metal ridge with a copper strip that is printed on a thin dielectric laminate. In this case, the configuration of the bed of nails conforms a completely regular periodic pattern which offers the possibility of its reuse by placing different printed circuits operating in the frequency band that is supported. Evidently, this leads to advantage in terms of time and manufacturing cost. Nevertheless, in either case, there is a need to design transitions to connect these new guides to the measurement devices through standard transmission lines. This paper presents a Test-Fixture by introducing the design of a transition from standard waveguide to suspended-strip gapwaveguide (SSGW). This provides a simple circuit assembly system for measurements on Ka-band of prototypes without soldering.

\section{TEST-FIXTURE SYSTEM}

The aim of this work was to design a Test-Fixture to measure devices made in SSGW without soldering. The measurement ports used were rectangular standard waveguides WR28 in order to cover the frequency band from 26.5 to $40 \mathrm{GHz}$. To connect the rectangular waveguide with the suspended strip, a rectangular coaxial (RCx) manufactured with two flexible dielectric substrates is proposed. These two substrates allow the suspended-strip to be captured like a sandwich.

\section{A. Rectangular coaxial}

A rectangular coaxial was designed using a woven reinforced PTFE substrate (Neltec NY9220) with permittivity of epsilon $_{r n}=2.2$ and losses of $\tan \delta=0.0009(10 \mathrm{GHz})$. The height of the dielectric was $h \_$diel $=508 \mu \mathrm{m}$ and the copper thickness was $t_{-}$copper $=35 \mu \mathrm{m}$. Fig. 1(a) shows the transversal section of the RCx. The height was determined by $h \_R C x=3 t \_c o p p e r+2 h \_d i e l=1.121 \mathrm{~mm}$. A width $w_{-} R C x=2 \mathrm{~mm}$ was chosen to avoid higher-order modes (cut-off frequency of the first higher-order mode $=50$ $\mathrm{GHz}$ ). Using the HFSS simulator, a width of the central strip $w \_s t r i p \_R C x=0.77 \mathrm{~mm}$ was chosen to get a characteristic impedance $Z_{o}=50 \Omega$. These dimensions together with the dielectric breakdown of the used substrate can bear a maximum RF power of about $38 \mathrm{~kW}$.
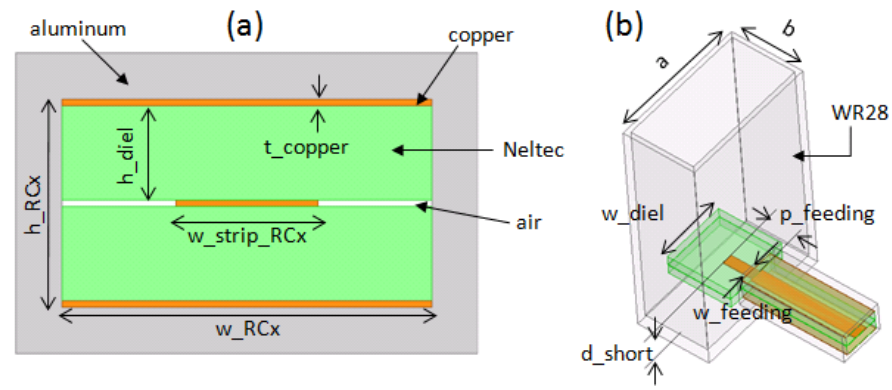

Fig. 1. (a)-Rectangular Coaxial. (b)-Rectangular Coaxial to Rectangular Waveguide transition.

\section{B. Transition design}

There are numerous works that design transitions between the microstrip line and the rectangular waveguide where probes are transverse to the propagation direction of the waveguide [6], [7]. This type of transition has been chosen in this work in order to simplify the connection with the suspended strip. The transition proposed here uses the central strip of the coaxial to feed the rectangular waveguide from its wide side (Fig. 1(b)). Beginning with the proposed values in other works ([6], [7]), and after applying an optimization process, the following relations were found: d_short = $0.15 \lambda_{g}=1.7 \mathrm{~mm} ; p_{\_}$feeding $=0.46 \mathrm{~b}=1.67 \mathrm{~mm}$; $w \_$feeding $=0.08 a=0.57 \mathrm{~mm}$. With these values a VSWR less than 1.3 was achieved between 26.5 and $40 \mathrm{GHz}$. A width of the two dielectric substrates, $w_{-}$diel $=3 \mathrm{~mm}$, higher than $w_{-} R C x$, was used to fit them inside the metal structure. This dimension does not affect the level of matching.

\section{Suspended-Strip Gap-Waveguide for Ka-band}

A bed of rectangular nails was selected for the design of the Suspended-Strip Gap-Waveguide (SSGW). A maximum band gap $(25-50 \mathrm{GHz})$ is achieved choosing a height of nail 
$h \_n a i l=1 / 4 \lambda=2 \mathrm{~mm}$, a period between nails $p \_n a i l=$ $3 / 8 \lambda=3 \mathrm{~mm}$, a width of $w \_n a i l=2 / 5 \mathrm{p} \_n a i l=1.2 \mathrm{~mm}$, and a gap of air $h \_$air $=1 / 20 \lambda=0.4 \mathrm{~mm}$, being $\lambda$ the wavelength in air at the central frequency $(f=37.5 \mathrm{GHz})$. The substrate used was Pyralux AP9121R of DuPont, with a dielectric thickness of $t \_p y r a l u x=50 \mu \mathrm{m}$ with permittivity of epsilon $_{r p}=3.6$ and a gold-plated copper thickness of $t \_g o l d \_p l a t e d \_c o p p e r=35 \mu \mathrm{m}$. Fig. 2 shows the structure of the SSGW. A maximum RF power of around $6.0 \mathrm{~kW}$ is allowed with these dimensions, for a typical suspended strip $\left(w \_s s g w=1.4 \mathrm{~mm}\right)$ and the dielectric breakdown of air. It is important to note that when the width of the strip on the substrate, $w \_s s g w$, is comparable to the period between nails, $p \_n a i l$, the impedance of the line is sensitive to the relative position of the strip over the nails [5]. One way to solve this is to increase the density of nails (doing period $p \_$nail smaller) at the cost of having a narrower band gap.

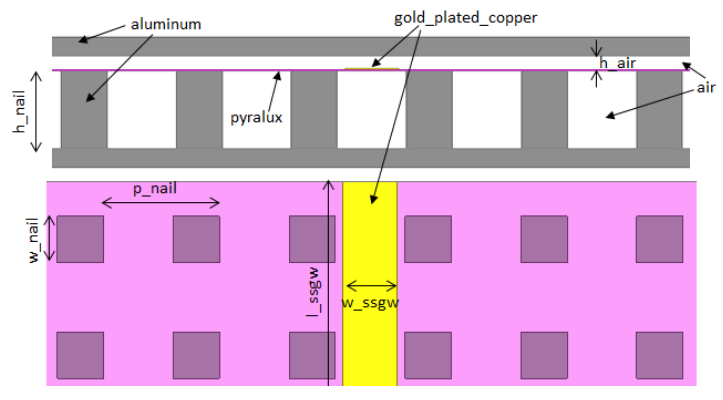

Fig. 2. Suspended-Strip Gap-Waveguide (SSGW).

\section{Test-Fixture Structure}

Once the transition between WR28 to RCx and also the SSGW was designed, a system to measure devices up to 4 ports was developed. Fig. 3 shows schematically the parts that make up one of the ports. The length of the RCx, $l_{-} R C x=10 \mathrm{~mm}$, was chosen to prevent the WR28 flange from overlapping the area of the SSGW (an important detail for the TRL kit). With this length, the higher-order modes of the RCx have more than $60 \mathrm{~dB}$ of attenuation.

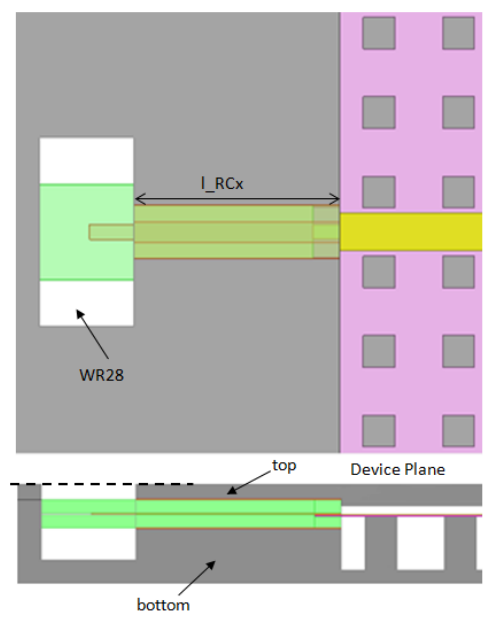

Fig. 3. Detail of a Test-Fixture port.

\section{MANUFACTURING AND MEASURE}

\section{A. TRL calibration kit}

A TRL calibration kit was designed with the aim of de-embedding the effect of the WR28-RCx transition. The elements of this kit were chosen to fix the reference plane just at the junction between the suspended strip and the RCx. The THRU is a combination of two WR28-RCx transitions and a RCx with $Z o=50 \Omega$ of length $l_{-} T H R U=2 \times$ $l_{-} R C x=20 \mathrm{~mm}$. The LINE is similar, but it adds a length of $\lambda_{R C x} / 4=1.37 \mathrm{~mm}$ at $f_{o}=36.85 \mathrm{GHz}$, which is the frequency of interest for subsequent circuits. The REFLECT is equal to the THRU but includes a via hole connecting the two grounds and the strip of the substrates in the middle. Fig. 4 shows the three elements of the kit.

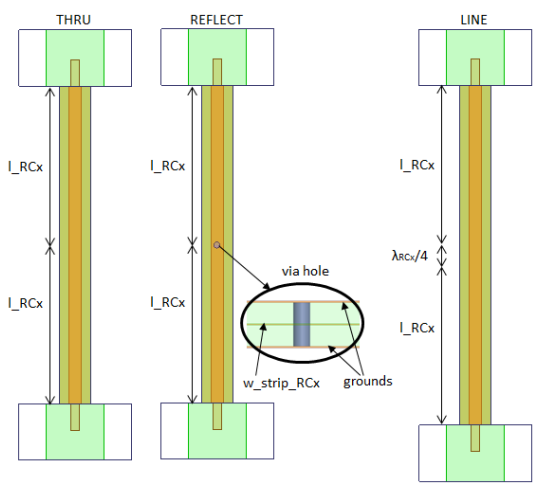

Fig. 4. TRL calibration kit.

\section{B. Measurement repeatability}

To assess the reliability of the assembly system that is designed to take measurements, it is important to know the repeatability of the process. This will indicate whether the measure is perfectly consistent and repeatable. To carry out the study, the results of a set of ten measures of the same suspended-strip waveguide line were collected. The suspended-strip waveguide line selected for the study had a width of $w \_s s g w=1.4 \mathrm{~mm}$ and a length of $l_{-} s s g w=$ $48 \mathrm{~mm}$. The line was assembled and disassembled for each of the measurements. Fig. 5 and Fig. 6 show the amplitudes and phases of $S_{11}$ and $S_{21}$ for the ten measurements.

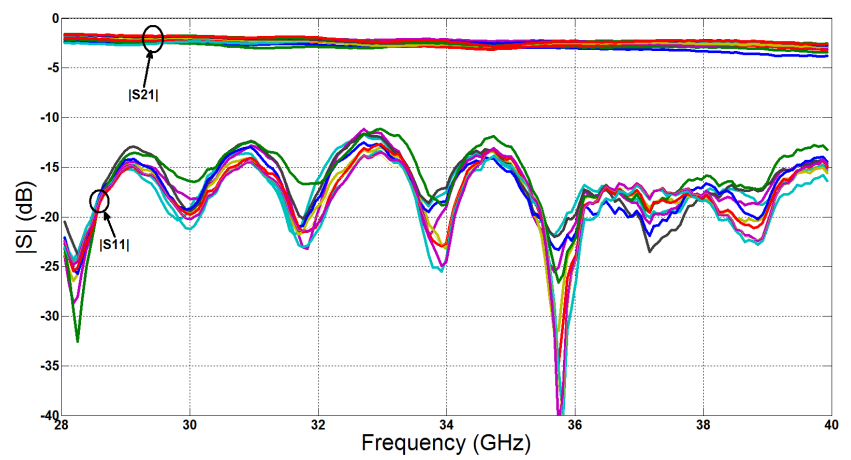

Fig. 5. Amplitudes of $S_{11}$ and $S_{21}$ for ten measurements.

Fig. 5 and Fig. 6 show that by calculating the mean of the scattering parameter $\mu\left(s_{m}\right)$ for each frequency and assuming that the mean of the error is approximately zero, $\mu($ error $) \approx 0$, the complex error for each frequency is error $=s_{m}-\mu\left(s_{m}\right)$. In the case of $S_{21}$, with higher values, the mean of the amplitude of this error is quite independent of frequency. The mean value in frequency is $\mu\left(\mid\right.$ error_ $\left.S_{21} \mid\right)=$ 0.032 , with a standard deviation of $\sigma\left(\left|e r r o r \_S_{21}\right|\right)=0.017$. For the case of $S_{11}$, with lower values, the mean of the 


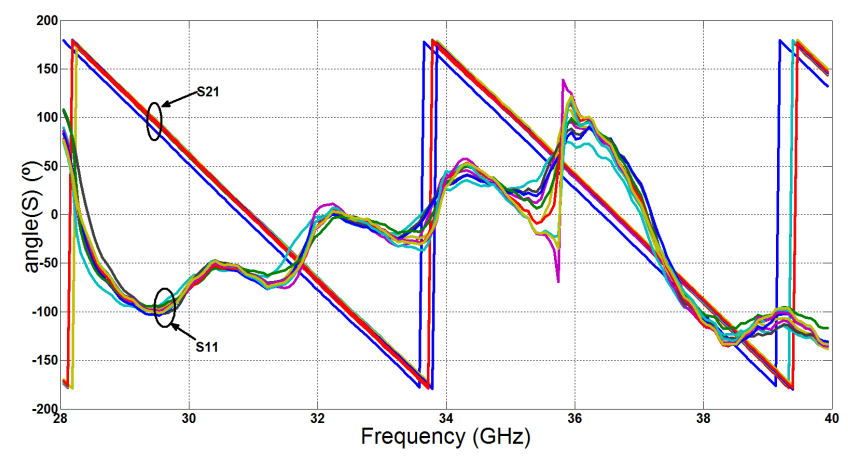

Fig. 6. Phases of $S_{11}$ and $S_{21}$ for ten measurements.

amplitude of the error is more dependent on the frequency. The mean value in frequency is $\mu\left(\mid\right.$ error_ $\left.S_{11} \mid\right)=0.020$, with a standard deviation of $\sigma\left(\mid\right.$ error_$\left.S_{11} \mid\right)=0.012$. The phase error shows considerable dispersion, but with a mean value of about $0^{\circ}$. Fig. 7 shows the percentage of the mean of the error amplitude over the mean of the measured scattering parameter amplitude versus the mean of the measured scattering parameter amplitude. Note that the device used in the measurement presents $\left|S_{11}\right|$ values (o symbol) that are lower than $-12 \mathrm{~dB}$ and $\left|S_{21}\right|$ values ( $\square$ symbol) that are around -2 $\mathrm{dB}$. The continuous line represents the theoretical result with $\mid$ error $\mid=0.032$. It is evident that the smaller the scattering parameter to measure, the more critical the error.

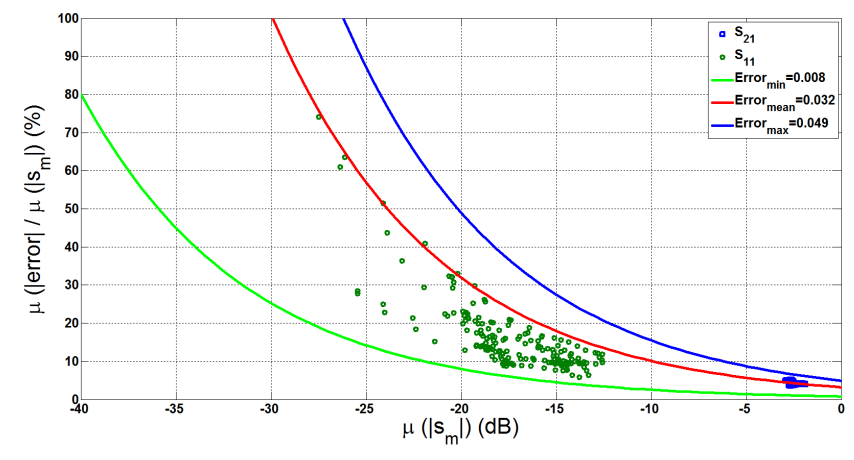

Fig. 7. Percentage of the |error|.

For an error of 0.032, Table I shows the theoretical maximum uncertainty value depending on the scattering parameter. These values can be considered to be acceptable for the aim of the Test-Fixture.

TABLE I

SCATTERING PARAMETER UNCERTAINTY.

\begin{tabular}{|c|c|c|c|c|c|c|}
\hline $\mathrm{S}(\mathrm{dB})$ & 0 & -5 & -10 & -15 & -20 & -25 \\
\hline$\Delta \mathrm{S}(\mathrm{dB})$ & 0.28 & 0.51 & 0.93 & 1.72 & 3.35 & 7.31 \\
\hline
\end{tabular}

\section{Prototyping}

Some circuits were made to check the feasibility of the Test-Fixture system that we designed. Both the simulated and measured results of an unbalanced three-way power divider are shown below. The simulations are referenced to the RCx on the same reference plane that the TRL calibration. Table II shows the results of the $\mathrm{S}$-parameter simulations and the measures of the three-way power divider. A good agreement between simulation and measurement is observed.
TABLE II

Three-WAy PoWer Divider. Simulated VS. MEASURED S-PARAMETERS AT $36.85 \mathrm{GHz}$.

\begin{tabular}{|c|c|c|c|c|}
\hline DIV & $S_{11}(\mathrm{~dB})$ & $S_{21}(\mathrm{~dB})$ & $S_{31}(\mathrm{~dB})$ & $S_{41}(\mathrm{~dB})$ \\
\hline Simulated & -13.64 & -9.33 & -9.33 & -4.30 \\
\hline Measured & -14.03 & -9.44 & -9.30 & -4.56 \\
\hline
\end{tabular}

Fig. 8 shows the Test-Fixture prototype with the three-way power divider assembled.

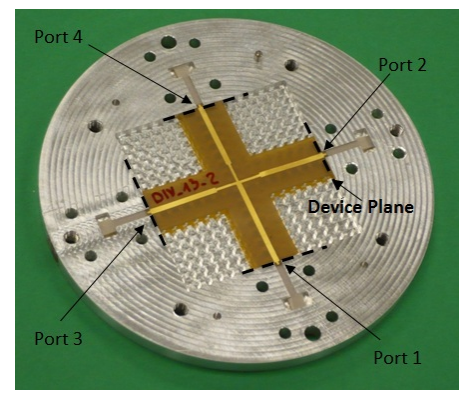

Fig. 8. Test-fixture Prototype. Three-Way Power Divider assembled.

\section{CONCLUSIONS}

In the last few years, research in Gap-Waveguide technology has been developed to replace conventional packaging techniques. SSGW could be a good way to design and manufacture prototypes quickly and at low cost. In this paper, a Test-Fixture system for these kinds of prototypes on Kaband has been presented. A TRL calibration kit has been designed to ensure the reliability of the S-parameter measures of the SSGW. Moreover, it has been demonstrated in this work that the assembly and disassembly of circuits with the rectangular standard waveguide-to-rectangular coaxial transition is consistent and repeatable. Finally, a fairly good agreement between simulation and measurement has been observed.

\section{ACKNOWLEDGEMENTS}

This work was mainly supported by the Spanish Ministerio de Ciencia e Innovacion under projects TEC2010-20841-C0401 and CSD2008-00068.

\section{REFERENCES}

[1] P. S. Kildal, "Three metamaterial-based gap waveguides between parallel metal plates for $\mathrm{mm} / \mathrm{submm}$ waves" 3rd European Conference on Antennas and Propagation, EuCAP 2009, pp. 28-32.

[2] P. S. Kildal, E. Alfonso, A. Valero-Nogueira, and E. Rajo-Iglesias, "Local metamaterial-based waveguides in gaps between parallel metal plates" IEEE Antennas and Wireless Propagation Letters, vol. 8, pp. 84-87, 2009.

[3] E. Alfonso, M. Baquero, P. S. Kildal, A. Valero-Nogueira, E. RajoIglesias and J. I. Herranz, "Design of Microwave Circuits in Ridge-Gap Waveguide Technology" Technical report, IEEE MTT 2010 International Microwave Symposium, May 2010.

[4] P. S. Kildal, E. Rajo-Iglesias, E. Pucci, A. U. Zaman and A. Kishk, "Losses in ridge gap waveguide compared with rectangular waveguides and microstrip transmission lines" Technical report, 4th European Conference in Antennas and Propagation, Apr. 2010.

[5] A. Valero-Nogueira, M. Baquero, J. I. Herranz, J. Domenech, E. Alfonso and A. Vila, "Gap waveguides using a suspended strip on a bed of nails" IEEE Antennas and Wireless Propagation Letters, vol. 10, pp. 1006-1009. 2011.

[6] Leong Yoke-Choy, and S. Weinreb, "Full Band Waveguide-to-Microstrip Probe Transitions " IEEE MTT-S Int. Microwave Symp. Dig., vol. 4, pp. 1435 1438, June 1999.

[7] C. Risacher, V. Vassilev, A. Pavolotsky, and V. Belitsky, "Waveguideto-microstrip transition with integrated bias-T" IEEE Microw. Wireless Compon. Lett., vol. 13, no. 7, pp. 262-264, Jul. 2003. 